\title{
Nonpegylated liposomal doxorubicin combination regimen in patients with diffuse large B-cell lymphoma and cardiac comorbidity. Results of the HEART01 phase II trial conducted by the Fondazione Italiana Linfomi
}

\author{
Stefano Luminari ${ }^{1,2}$ | Elda Viel ${ }^{3}$ | Andrés José Maria Ferreri ${ }^{4}$ | Francesco Zaja ${ }^{5}$ | \\ Emanuela Chimienti ${ }^{6}$ | Gerardo Musuraca ${ }^{7}$ | Alessandra Tucci $^{8}$ | Monica Balzarotti ${ }^{9}$ | \\ Monica Tani ${ }^{10}$ | Francesca Salvi ${ }^{11}$ | Emanuela A. Pesce ${ }^{12}$ (D) | Angela Ferrari ${ }^{1}$ | \\ Anna M. Liberati ${ }^{13}$ | Antonio Spadea ${ }^{14}$ | Dario Marino ${ }^{15}$ | Maria Bruno-Ventre ${ }^{4}$ | \\ Stefano Volpetti ${ }^{5}$ | Chiara Bottelli ${ }^{8}$ | Elena Ravaioli ${ }^{6}$ | Francesco Merli ${ }^{1}$ | Michele Spina ${ }^{6}$ \\ ${ }^{1}$ Hematology, Arcispedale Santa Maria Nuova, Istituto di Ricovero e Cura a Carattere Scientifico, Reggio Emilia, Italy \\ ${ }^{2}$ Department of Diagnostic, Clinic and Public Health Medicine, University of Modena and Reggio Emilia, Modena, Italy \\ ${ }^{3}$ Cardiology Unit, National Cancer Center, Istituto di Ricovero e Cura a Carattere Scientifico, Aviano, Pordenone, Italy \\ ${ }^{4}$ Unit of Lymphoid Malignancies, IRCCS San Raffaele Scientific Institute, Milan, Italy \\ ${ }^{5}$ Haematology Section, DISM, Azienda Sanitaria Universitaria Integrata S. M. Misericordia, Udine, Italy \\ ${ }^{6}$ Division of Medical Oncology A, National Cancer Center, Istituto di Ricovero e Cura a Carattere Scientifico, Aviano, Pordenone, Italy \\ ${ }^{7}$ Department of Hematology, Istituto Scientifico Romagnolo per Studio e Cura dei Tumori, Meldola, Forlì-Cesena, Italy \\ ${ }^{8}$ Division of Hematology, Spedali Civili, Brescia, Italy \\ ${ }^{9}$ Hematology Unit, Humanitas Cancer Center, Rozzano, Milan, Italy \\ ${ }^{10}$ Hematology Unit, Santa Maria delle Croci Hospital, Ravenna, Italy \\ ${ }^{11}$ Department of Hematology, SS Antonio E Biagio E Cesare Arrigo Hospital, Alessandria, Italy \\ ${ }^{12}$ Fondazione Italiana Linfomi Onlus, Italy \\ ${ }^{13}$ Oncohematology, AO S. Maria di Terni, Terni, Italy \\ ${ }^{14}$ Hematology and Stem Cell Transplant Unit, Regina Elena National Cancer Institute, Rome, Italy \\ ${ }^{15}$ Division of Medical Oncology 1, Istituto Oncologico Veneto-IRCCS, Padova, Italy
}

Correspondence

Prof Stefano Luminari, University of Modena and Reggio Emilia, Hematology Unit, Department of Oncology, Arcispedale Santa Maria Nuova, Istituto di Ricovero e Cura a Carattere Scientifico, Viale Risorgimento 80, Reggio Emilia 42123, Italy.

Email: luminari.stefano@asmn.re.it; stefano.luminari@unimore.it

Funding information

Teva Pharmaceutical Industries

Clinical trial registration: NCT01009970

\begin{abstract}
The purpose of this phase 2, multicenter study was to determine the activity and safety of nonpegylated liposomal doxorubicin as part of "RCOMP" combination in patients with diffuse large B-cell lymphoma and coexisting cardiac disorders. The study was conducted using a Bayesian continuing assessment method using complete remission rate and rate of cardiac events as study endpoints. Between November 2009 and October 2011, 50 evaluable patients were enrolled (median age, 76 years). Median baseline left ventricular ejection fraction (LVEF) was $60 \%$. Ischemic cardiopathy was the most frequent preexisting cardiac disorder (35\%), followed by atrial fibrillation (15\%), left ventricular hypertrophy (13\%), and baseline LVEF $<50 \%(12 \%)$. Based on the intent to treat analysis, overall response rate was $72 \%$, including 28 patients in complete remission (complete remission rate, 56\%), and 8 in partial remission (16\%). At the end of treatment, grades 3 to 4 cardiac events were observed in 6 patients. No significant modifications from baseline values of LVEF were observed during treatment and follow-up.
\end{abstract}


Nonpegylated liposomal doxorubicin instead of doxorubicin in the R-CHOP (rituximab, cyclophosphamide, doxorubicin, vincristine, and prednisone) regimen is a feasible option for patients with diffuse large B-cell lymphoma presenting with concomitant cardiac disorders.

\section{KEYWORDS}

cardiotoxicity, diffuse large B-cell lymphoma, non-pegylated liposomal doxorubicin

\section{1 | INTRODUCTION}

Diffuse large B-cell lymphoma (DLBCL) is the most common subtype of non-Hodgkin lymphomas, ${ }^{1}$ typically affects elderly patients and can be cured in $60 \%$ to $70 \%$ of cases with the standard $\mathrm{R}-\mathrm{CHOP}$ immunochemotherapy. ${ }^{2,3}$ Conventional anthracyclines are the active backbone of standard R-CHOP, but cardiotoxicity related to the cumulative dose may contraindicate or limit their use especially in patients with preexisting or concomitant cardiac disorders. ${ }^{4}$ It has also been shown that cardiotoxicity was the predominant late chemotherapyrelated complication in long-term survivors with aggressive lymphoma. ${ }^{5}$ However, cardiotoxicity data in patients with DLBCL are fragmentary and derive from relatively small studies, since patients older than 65, whose age-associated comorbidities may confer a higher risk of anthracycline cardiotoxicity, are often excluded from clinical trials. ${ }^{6}$

Strategies to improve the cardiac safety of conventional anthracyclines include alterations of dosing schedules to modify pharmacokinetics, ${ }^{7}$ use of other conventional anthracyclines that may be less cardiotoxic, administration of cardioprotective agents, ${ }^{8}$ and administration of anthracyclines in liposome-encapsulated. In particular, the use of nonpegylated liposomal doxorubicin (NPLD) is associated with reduced myelosuppression, ${ }^{9}$ reduced gastrointestinal toxicity, ${ }^{10}$ and a reduced risk of cardiotoxicity ${ }^{11}$ compared with standard formulations. A phase III randomized trial that compared NLPD and cyclophosphamide with doxorubicin and cyclophosphamide in patients with metastatic breast cancer revealed significantly reduced cardiotoxicity in the NLPD arm, without reducing the efficacy of therapy. ${ }^{12}$ The toxicity and activity of NPLD when substituted for conventional doxorubicin in the CHOP regimen (so-called "R-COMP" regimen) have been evaluated also in the treatment of patients with newly diagnosed aggressive non-Hodgkin lymphomas, and the combination has been found to be an effective and relatively well tolerated regimen, ${ }^{13,14}$ even in patients with concurrent cardiac diseases or prior anthracyclines exposure. ${ }^{15}$ On this background, we designed a multicenter, single-arm phase II trial addressing the activity and safety of the R-COMP combination as upfront treatment for patients with DLBCL who had concomitant or pre-existing cardiovascular disorders (CDs).

\section{2 | METHODS}

\subsection{Study design and eligibility criteria}

This is an open label, single-arm, multicenter phase II trial evaluating the safety and efficacy of replacing doxorubicin with NLPD in standard $\mathrm{R}-\mathrm{CHOP}$ in patients with newly diagnosed $\mathrm{DLBCL}$ and concomitant or preexisting cardiac disorders. Selection criteria were (1) biopsy-confirmed CD2O + DLBCL or follicular grade III b; (2) age $\geq 18$ years; (3) at least one of the following preexisting or concomitant CDs: left ventricular ejection fraction (LVEF) $<50 \%$, left ventricular hypertrophy (septal wall and/or posterior wall thickness $>1.2 \mathrm{~cm}$ ), moderate to severe high blood pressure not controlled by therapy, documented ischemic heart disease, significant ventricular arrhythmias (score 3 according to the Lown grading system ${ }^{16}$ ), chronic atrial fibrillation, pulmonary hypertension (mean estimating Pulmonary Artery Pressure (mPAP) > $45 \mathrm{mmHg}$ ), moderate to severe mitral valve disease, and moderate aortic valve disease (mean pressure gradient, $20-40 \mathrm{~mm}$ $\mathrm{Hg}$ ). The Baseline CDs were categorized into 3 groups: electrical, including heart rhythm disorders (arrhythmias and atrial fibrillation); circulatory, including high blood pressure, ischemic heart disease, and pulmonary hypertension; structural, including reduced LVEF, ventricular hypertrophy and valve disease. All patients were evaluated by Comprehensive Geriatric Assessment, including Activities of Daily Living, Instrumental Activities of Daily Living, Cumulative Illness Rating Scale. Activities of Daily Living $=6$, Instrumental Activities of Daily Living $\geq 4$, a maximum of 2 grade- 2 extra-cardiac comorbidities and absence of grade- 3 extra-cardiac comorbidities, and no geriatric syndrome were required for patients aged 70 years or older. Other inclusion criteria were serum creatinine levels $<2.5 \mathrm{mg} / \mathrm{dL}$, bilirubin $\leq$ twice upper the normal range, negative serology for hepatitis $B$, hepatitis $C$, and HIV. Any stage and IPI score were considered.

\section{2 | Evaluations}

Baseline assessment included disease history, B symptoms, physical examination, laboratory assessments, contrast enhanced computed tomography (CT) scan, electrocardiogram and cardiac examination, bidimensional echocardiogram (2D-ECD), and bone marrow biopsy. ${ }^{17}$ Fluorodeoxyglucose-positron emission tomography was recommended at baseline. Cardiac function was assessed before treatment start and was monitored after 3 cycles, at the end of therapy and during follow-up with 12-leads electrocardiogram and with bi-dimensional echocardiogram. Bi-dimensional echocardiogram had to be performed locally at each referring center; no routine assessment of serum cardiac troponin or B-type natriuretic peptide was required by the study protocol. Final response had to be assessed in all evaluable patients by contrast enhanced CT, positron emission tomography-CT scans, and every procedure positive at baseline. During follow-up, patients were clinically evaluated every 3 months for 2 years and then every 6 months for 1 year, with restaging CT imaging at months 6, 12, 24, and 36 from the end of the treatment.

This study was conducted according to the Good Clinical Practice guidelines and the October 2000 revision of the Declaration of 
Helsinki. The study protocol was approved by the ethic committees according to local rules. All patients gave their written informed consent to participate before study entry. Patient registration and data collection were realized using a dedicated web platform.

\section{3 | Treatment}

The R-COMP regimen consisted of a standard R-CHOP, replacing doxorubicin with the same doses of NPLD (cyclophosphamide $750 \mathrm{mg} / \mathrm{m}^{2}$, day 1 ; vincristine $1.4 \mathrm{mg} / \mathrm{m}^{2}$ maximum dose of $2 \mathrm{mg}$, day 1; NPLD $50 \mathrm{mg} / \mathrm{m}^{2}$, day 1; and prednisone $100 \mathrm{mg} /$ day, days 15; rituximab $375 \mathrm{mg} / \mathrm{m}^{2}$, day 3 of cycle 1 and day 1 of subsequent cycles). The NPLD (Myocet) was provided for free by the manufacturer. The R-COMP had to be delivered on an outpatient basis every 3 weeks for 4 cycles in patients with stage I to II non-Bulky disease and for 6 cycles in all other patients.

Patients were treated with supportive medications, including prophylactic antibiotics and antiemetic agents, according to physician's discretion. Granulocyte colony-stimulating factor was administered as per institutional guidelines. In case of peripheral neuropathy, vincristine doses could be reduced or withdrawn. Patients at risk of central nervous system relapse received intrathecal doses of $12 \mathrm{mg}$ methotrexate on day 1 of each cycle. ${ }^{18}$

The R-COMP was interrupted in case of grades 3 to 4 nonhematologic toxicity causing treatment delay of more than 3 weeks or for LVEF decrease of $20 \%$ from baseline or absolute 10 points in LEVF from baseline.

\section{4 | Statistics}

The primary end points of this phase II study were the complete remission rate (CRR) and the rate of cardiac events (CEs). Responses were assessed using the 2007 International Harmonization Project criteria. $^{17}$

Cardiac events were defined as LVEF decrease $\geq 20 \%$ from baseline or absolute LVEF $<25 \%$ at the end of treatment or clinical evidence of heart failure. The coprimary end point analyses were performed for all eligible patients according to an intent to treat principle.

The study enrollment and monitoring was planned according to a Bayesian sequential analysis, considering a reference CRR of $65 \%{ }^{19}$ and CEs rate of $15 \%{ }^{20}$ We planned to recruit a total of 55 patients, also considering a dropout rate of about $10 \%$ to reach 50 eligible patients. The a priori probability of CR and CEs were modeled by a beta distribution, with parameters $\mathrm{B}(1.32 ; 0.68)$ for efficacy and $\mathrm{B}(0.32 ; 1.68)$ for the safety. The trial was monitored by cohorts of 10 patients, and the study had to be interrupted if the observed response rate was lower than the reference $\mathrm{CR}$, with a posterior probability greater than $95 \%$ (or more responsive with a posterior distribution lower than 0.05) or if the observed rate of CEs was higher than the reference, with a posterior probability greater than 0.95 .

The final CRR and CEs were reported with the exact ClopperPearson confidence interval limits (Cls) and as posterior 95\% credible interval. The paired comparison between baseline and end of treatment LVEF was calculated using the Wilcoxon matched pairs signedrank test.
The secondary end points were overall response rate (ORR), progression-free survival (PFS), and overall survival (OS).

Adverse events were categorized and graded according to the National Cancer Institute Common Terminology Criteria for Adverse Events (version 3.0).

\section{3 | RESULTS}

\section{1 | Patients}

Between November 2009 and October 2011, 51 patients were enrolled. One patient was subsequently excluded due to a violation of the inclusion criteria. Sixty CDs were identified at baseline; 2 and 3 concomitant CDs were described in 4 and 3 patients respectively. Baseline patients characteristics are shown in Table 1.

TABLE 1 Patients characteristics

\begin{tabular}{|c|c|c|c|}
\hline Variable & $\mathrm{N}$ & $\%$ & Missing $\mathrm{N}(\%)$ \\
\hline \multicolumn{4}{|l|}{ Age } \\
\hline Median & 76 & & \\
\hline Range & $53-90$ & & - \\
\hline$>60$ & 47 & 94 & \\
\hline Sex, M & 35 & 70 & - \\
\hline \multicolumn{4}{|l|}{ Stage } \\
\hline$|-I|$ & 19 & 38 & - \\
\hline III-IV & 31 & 62 & \\
\hline $\mathrm{PS}>1$ & 7 & 14 & - \\
\hline $\mathrm{LDH}>\mathrm{UNL}$ & 23 & 51 & $5(10)$ \\
\hline ENS > 1 & 5 & 10 & - \\
\hline Bulky & 5 & 10 & $1(2)$ \\
\hline \multicolumn{4}{|l|}{ IPI } \\
\hline $0-1$ & 11 & 24 & \\
\hline 2 & 16 & 26 & $5(10)$ \\
\hline $3-5$ & 18 & 40 & \\
\hline \multicolumn{4}{|l|}{ Cardiac disorders } \\
\hline Ischemic cardiopathy & 21 & 35 & \\
\hline Atrial fibrillation & 9 & 15 & \\
\hline Left ventricular hypertrophy & 8 & 13 & \\
\hline LVEF $<50 \%$ & 7 & 12 & \\
\hline Ventricular arrhythmia & 5 & 8 & - \\
\hline Moderate/severe mitral valve disease & 3 & 5 & \\
\hline Moderate aortic valve disease & 3 & 5 & \\
\hline Pulmonary hypertension & 2 & 3 & \\
\hline Uncontrolled hypertension & 2 & 3 & \\
\hline Altered ECG & 27 & 59 & $4(8)$ \\
\hline \multicolumn{4}{|l|}{ LVEF } \\
\hline Median & 60 & - & $3(6)$ \\
\hline IQR & 12 & & \\
\hline
\end{tabular}

Abbreviations: ECG, echocardiogram; ENS, extra nodal site; IPI, international prognostic index; IQR, interquartile range; $L D H$, lactate dehydrogenase; LVEF, left ventricular ejection fraction; $M$, male; PS, performance status; UNL, upper normal value.

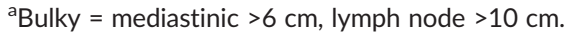




\subsection{Feasibility and efficacy}

The R-COMP was started in all 50 patients; 49 patients (98\%) received at least 3 cycles and 38 (76\%) completed the treatment receiving 6 courses $(N=30)$, or 4 courses of R-COMP $(N=8)$ if they had a localized disease at stage I to II according to trial protocol. Treatment was interrupted prematurely in 12 cases due to adverse events $(N=10)$, progressive disease $(N=1)$, or patient decision $(N=1)$.

Overall, 261 cycles of R-COMP were delivered. All but 68 cycles were administered on time: median delay was 3 days (range 1 to 28). Dose reductions were adopted in 22 patients and in 68 cycles. The NPLD was reduced in 13 cases and in 37 cycles. The mean calculated dose intensity for all cycles of cyclophosphamide, NPLD, vincristine, and rituximab were $92.2 \%, 90.6 \%, 89.6 \%$, and $95.6 \%$, respectively.

Based on the intention to treat analysis, 28 patients achieved a CR at the end of therapy ( $56 \% ; 95 \% \mathrm{Cl}, 41 \%-70 \%)$, and 36 patients had an objective response (ORR $=72 \% ; 95 \% \mathrm{Cl}, 58 \%$ to $84 \%$ ) (Table 2$)$. As requested by the sequential Bayesian monitoring, the $\mathrm{CR}$ rate never fell outside activity boundaries (Figure $1 a$ in the Supporting information), thus the study achieved its main planned activity result.

The median follow-up was 33 months (range 1-61). Regarding the definition of PFS, 30 events were reported including 11 progressions, 12 relapses, and 7 deaths for causes unrelated with lymphoma. Median PFS was 17 months (95\% Cl; 9-NA months). Progression-free survival at 3 years was $38 \%(95 \% \mathrm{Cl}$; $24 \%-51 \%)$ (Figure $1 \mathrm{~A})$.

Overall, 22 patients died, 6 during the treatment or within 3 months from treatment completion ( 2 due to lymphoma progression and one each due to acute renal failure, acute liver failure, cerebral hemorrhage, and pneumonia), and 16 during the follow-up phase (7 due to lymphoma progression, 2 each due to heart failure and severe infection, one each due to respiratory failure, cachexia not related with lymphoma, and lung cancer; in 2 cases, the cause of death was unknown). Median age of patients died for causes unrelated to lymphoma progression was 78 years (range 70-84). As a result, OS at 3 years was $50 \%$ (95\%Cl; 33\%-65\%) (Figure $1 \mathrm{~B})$.

\section{3 | Safety}

Safety analysis was available for all 50 patients and for 248 cycles. Neutropenia was the most common hematologic event, with grades

TABLE 2 Summary of study end points $(N=50)$

\begin{tabular}{ccc} 
Response & N & $\%(95 \mathrm{Cl})$ \\
\hline CR & 28 & $56(41-70)$ \\
\hline PR & 8 & $16(4-29)$ \\
\hline ORR & 36 & $72(58-84)$ \\
\hline SD/PD & 10 & $20(10-34)$ \\
\hline NA/EW & 4 & $8(2-19)$ \\
\hline 3-yr survival & $\#$ events & $\%(95 \mathrm{Cl}$ \\
\hline OS & 22 & $50(34-65)$ \\
\hline PFS & 30 & $38(24-51)$ \\
\hline FFS & 36 & $27(15-40)$ \\
\hline
\end{tabular}

Abbreviations: $\mathrm{Cl}$, confidence interval; $\mathrm{CR}$, complete response; $\mathrm{EW}$, early withdrawal; FFS, failure free survival; NA, not assessed; ORR, overall response rate; OS, overall survival; $\mathrm{PD}$, progressive disease; PFS, progression free survival; PR, partial response; SD, stable disease.
3 to 4 toxicity occurring in $64 \%$ of patients. Excluding cardiac toxicity, grades 3 to 4 infections were the most common nonhematologic adverse events (6\%) (Table 3). Granulocyte colony-stimulating factor was used in 44 patients.

\subsection{Cardiac events}

Ten patients experienced $11 \mathrm{CEs}$ during treatment that were graded as 3 to 4 in 6 cases (12\%). Grades 3 to 4 CEs included 3 cases of LVEF reduction $\geq 20 \%$ from baseline, 1 case each of congestive heart failure, angina, and atrial fibrillation. In 1 case, LVEF decrease was also associated with an increase of troponin level (Table 4). Overall, study treatment was discontinued due to the occurrence of CEs in 6 cases, all occurred within the first 4 cycles ( 2 after cycle 3 , and 3 after cycle 4). No patient died as a consequence of a CE; the remaining 4 patients who experienced cardiac problems during study treatment continued with the R-COMP regimen, receiving an average of 5 courses (range 3-6) and achieving CR in 2. As requested by the sequential Bayesian monitoring, the CE rate never fell outside safety boundaries (as shown in Figure $1 \mathrm{~b}$ in the Supporting information), thus the study achieved the predefined safety results.

Two patients died for congestive heart failure during the follow-up at 11 and 28 months from the last administration of R-COMP, respectively.

Considering LVEF monitoring, this was evaluated in 37 patients with both assessments performed at baseline and end of therapy; in 13 cases, LVEF evaluation has not performed due to treatment interruption for toxicity (5) and for medical decision (2), due to unavailability of patient (4), progression of disease (1), and loss to follow-up (1). At least 1 assessment during follow-up was performed in 27 cases. We observed a slight but not significant decrease from baseline to the end of treatment of the median LVEF (measured reduction $-4.0 \%$, $P=.112$ ) that remains stable during follow-up (Figure 2). A significant drop of LVEF was observed in individual cases.

We also analyzed the $\mathrm{CE}$ rates according to pretreatment $\mathrm{CDs}$ and observed a trend of higher rate of CEs in the patients affected by electrical disorders (30\%) compared to the other 2 groups (circulatory and structural disorders, with $16 \%$ and $7 \%$ CEs respectively; $P=.227$ ). Three CEs ( 1 increased troponin, 2 LVEF drop $\geq 20 \%$ ) occurred among the 7 patients who were enrolled with a LVEF $<50 \%$.

\section{4 | DISCUSSION}

In the HEART01 study, we investigated the activity and the safety of the R-COMP regimen in which conventional doxorubicin was substituted with the same dose of NPLD for the treatment of patients with DLBCL who also had one or more concomitant or preexisting cardiac disorder. With 50 enrolled patients and with a median follow-up of 17 months, we were able to show a CR rate of 56\%, a 3-year PFS of $38 \%$ and a 3 -year OS of $50 \%$ with manageable adverse events that were mostly represented by severe neutropenia. Of note, the rate of CEs during therapy was $21 \%$ with a $10 \%$ rate of severe events but without any cardiac death during treatment. These activity and safety results fulfilled the initially planned study assumption and allow us to 


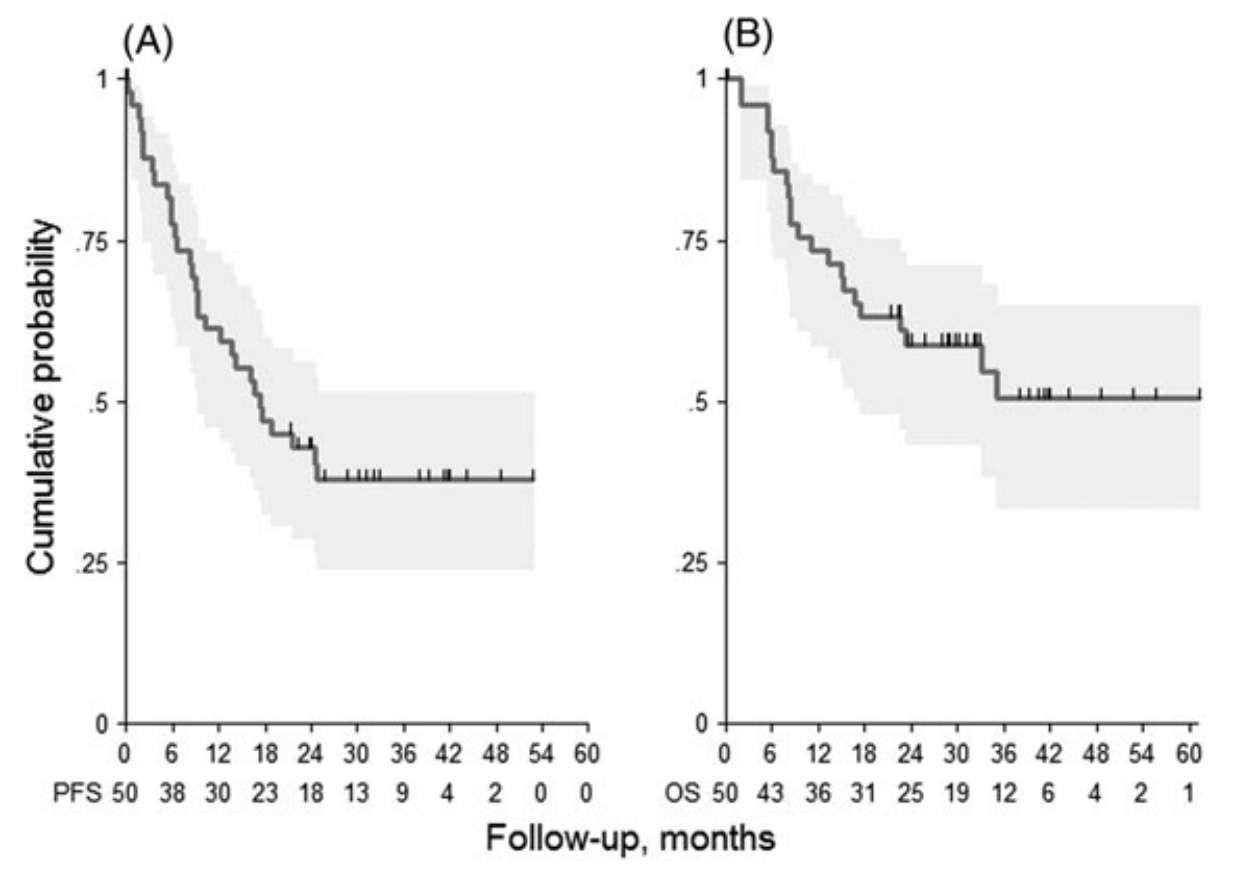

FIGURE 1 A, Progression free survival (PFS); B, overall survival (OS)

TABLE 3 Summary of clinical relevant adverse events occurred

\begin{tabular}{lcc} 
& \multicolumn{2}{c}{ Population (N = 50) } \\
\cline { 2 - 3 } Adverse event & Any grade (\%) & Grades 3-4 (\%) \\
\hline Blood and lymphatic system disorders & \\
\hline Neutropenia & 78 & 64 \\
\hline Anemia & 88 & 46 \\
\hline Thrombocytopenia & 58 & 8 \\
\hline Febrile neutropenia & 2 & 2 \\
\hline Gastrointestinal disorders & & \\
\hline Constipation & 6 & - \\
\hline Nausea & 6 & 2 \\
\hline Diarrhea & 2 & - \\
\hline Abdominal pain & 2 & - \\
\hline Vomiting & 4 & - \\
\hline Stomatitis & 4 & - \\
\hline Infections & 22 & 6 \\
\hline General disorders & & \\
\hline Pyrexia & 4 & - \\
\hline Asthenia & 10 & 2 \\
\hline Nervous system disorders & & 0 \\
\hline Paresthesia & 18 & \\
\hline & & \\
\hline
\end{tabular}

conclude this study with positive results and to consider R-COMP as a safe and active treatment option in patients with DLBCL and coexisting CDs.

Until now, NPLD has been evaluated in retrospective ${ }^{21,22}$ and in prospective studies ${ }^{23-27}$ not selecting the patients with preexisting heart disease. The NPLD activity and safety profile in patients with contraindication to the use of conventional anthracyclines including cardiac disorders have been assessed only in 1 study. ${ }^{15}$ More recently, 2 randomized studies compared the cardiotoxicity of NPLD with that
TABLE 4 Summary of cardiac events during treatment

\begin{tabular}{lcc} 
& \multicolumn{2}{c}{ Population (N = 50) } \\
\cline { 2 - 3 } Cardiac disorder & Grades 1-2, n (\%) & Grades 3-4, n (\%) \\
\hline Heart failure & $1(2)$ & $1(2)$ \\
LVEF drop $\geq 20 \%$ & $2(4)^{\mathrm{a}}$ & $3(6)$ \\
\hline Increased troponin & $2(4)$ & - \\
\hline Angina & - & $1(2)$ \\
Atrial fibrillation & - & $1(2)$ \\
Tot & $5(10)$ & $6(12)$ \\
\hline
\end{tabular}

Abbreviations: LVEF, left ventricular ejection fraction.

${ }^{\text {aAsymptomatic. }}$

of conventional doxorubicin in 2 independent series of patients with untreated DLBCL. ${ }^{28,29}$ Both studies included patients with normal cardiac function and were not able to show significant differences in the low observed rate of $\mathrm{CEs}$ between conventional doxorubicin and NPLD, also if safety signals were elevated in R-CHOP compared to R-COMP.

Of note, this prospective study evaluated the feasibility of a full dose chemoimmunotherapy regimen in a patient population that due to the presence of one or more CD would not have been considered eligible for a curative approach. The feasibility of the R-COMP regimen was confirmed with $76 \%$ of patients who were able to complete the planned therapy and with a very high mean dose intensity, also including NPLD. Of note, none of CEs that occurred during treatment resulted in patients' death. Our results should be compared with the study by Fields et al. that similarly included patients with coexisting CDs and substituted doxorubicin with gemcitabine within the R-CHOP chemotherapy backbone. ${ }^{30}$ Although response rates and survival were comparable between the 2 studies, Fields et al. apparently included patients with more severe coexisting CDs; this might explain the 


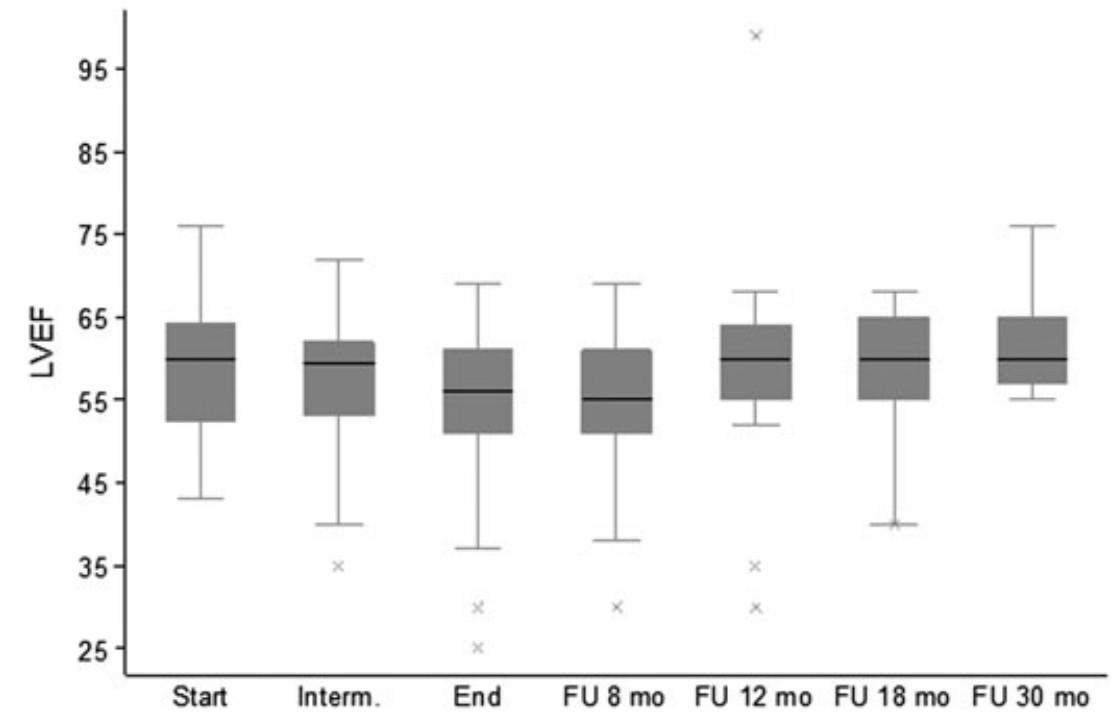

FIGURE 2 Left ventricular ejection fraction trend from baseline to end of follow up higher rates of severe CEs (16\%), and the 3 deaths occurred during treatment due to CEs. Also, if a formal comparison is not appropriate with other available clinical trials on $\mathrm{DLBCL},{ }^{2}$ our results look slightly worse if compared with a reference 3 -year OS of $70 \%$ for patients with DLBCL and with a similar age and confirm that patients with DLBCL and CDs as defined in our study or with contraindication to the use of anthracyclines represent a "hard to treat" population. ${ }^{30,31}$

In addition to the small sample size that is typical for a phase II study, our results could be influenced by the initial definition of the cardiac events that was based on a pragmatic approach and included most of the conditions that would have caused a reduction of the anthracycline dose if present. Considering the mechanism of anthracycline-induced cardiac toxicity, we acknowledge that the actual risk of doxorubicin induced cardiac toxicity can vary among patients with different cardiac conditions. In an exploratory analysis, we found that the CE rate was increasingly high comparing patients with electrical disorders to those with circulatory and structural disorders. In patients with structural disorders, the risk of experiencing CE is low. In contrast, in patient with low baseline LVEF, the CE rate is still high also if an NPLD is administered in place of conventional doxorubicin. Thus, the HEART01 study suggests that among patients with $C D$, the contraindication to the use of doxorubicin may change according to the quality of $C D$. In particular in patients at very high risk of $C E$, it is likely that the use of NPLD is not safe enough, and different strategies should be considered to preserve cardiac function and to allow the administration of oncologic therapy with curative intent. The use of doxorubicin-free regimens might represent a good option ${ }^{30,31}$ or, if doxorubicin containing regimens are used, prevention of anthracycline-induced cardiotoxicity could be considered before chemotherapy start. An accurate pretreatment cardiac evaluation could provide treatment of modifiable risks factors and optimize the cardiological therapy.

In conclusion, the HEART01 trial provides evidence of the feasibility and activity of the use of NPLD in patients with DLBCL and moderate/severe cardiac comorbidity. There are very few available studies with similar inclusion criteria, and patient with $\mathrm{CDs}$ are usually excluded from the large randomized trials. Nonetheless, patient with CC are frequently seen in daily practice, and based on currently available guidelines, no clear recommendation can be done, but the use of anthracyclines is usually vaguely contraindicated.

The explored strategy resulted in a feasible and active anthracycline-containing regimen, maintaining the curative intent of treatment in unfit, high-risk patients. This trial sets the reference activity and toxicity values for future studies willing to investigate new approaches in a similar setting.

\section{AUTHORS' DISCLOSURES OF POTENTIAL CONFLICTS OF INTEREST}

SL has served as consultant for Roche, Celgene, and Teva (Formerly Cephalon), has received an honoraria from Pfizer. FZ has received research funding from GSK, Novartis, and Celgene and has received honoraria from GSK, Novartis, Roche, Janssen, Takeda, Mundipharma, Gilead, and Celgene. MS has served on advisory board and on speakers' bureau, and he has received travel/accommodation from Teva (Formerly Cephalon). AJMF is a member of advisory boards of Acerta, Celgene, Gilead, Italfarmaco, and Mundipharma, received grants supporting the own clinical research from Celgene, and Mundipharma, and was invited speaker in meeting organized by Adienne, Gilead, Mundipharma, and Italfarmaco. All remaining authors have declared no conflicts of interest.

\section{ACKNOWLEDGEMENTS}

We thank all the patients who participated in this study and their supportive families. We also thank the referring physicians and the supporting staff at all the participating clinical sites. We thank all the principal investigators of the other sites involved in this study: Maura Brugiatelli (Messina), Salvatrice Mancuso (Palermo), Nicola Cascavilla (S. Giovanni Rotondo), and Graziella Pinotti (Varese).

\section{FUNDING}

This work was supported by Cephalon that provided the Fondazione Italiana Linfomi with NPLD, but was not involved in the study protocol, data acquisition, data analysis or the writing of the paper. There is no grant number. 


\section{AUTHORS' CONTRIBUTIONS}

EV, AT, FS, and MS designed the research study. All authors contributed to patient enrolment. SL analysed the data, performed the statistical analyses, and wrote the first draft of the article. All authors contributed to data interpretation, reviewed the draft, and approved the final version.

\section{REFERENCES}

1. Campo E, Swerdlow SH, Harris NL, Pileri S, Stein H, Jaffe ES. The 2008 WHO classification of lymphoid neoplasms and beyond: evolving concepts and practical applications. Blood. 2011;117(19):5019-5032. https://doi.org/10.1182/blood-2011-01-293050

2. Coiffier B, Lepage E, Briere J, et al. CHOP chemotherapy plus rituximab compared with $\mathrm{CHOP}$ alone in elderly patients with diffuse large-B-cell lymphoma. N Engl J Med. 2002;346(4):235-242.

3. Habermann TM, Weller EA, Morrison VA, et al. Rituximab-CHOP versus $\mathrm{CHOP}$ alone or with maintenance rituximab in older patients with diffuse large B-cell lymphoma. J Clin Oncol. 2006;24(19):3121-3127.

4. Lipshultz SE, Lipsitz SR, Mone SM, et al. Female sex and drug dose as risk factors for late cardiotoxic effects of doxorubicin therapy for childhood cancer. N Engl J Med. 1995;332(26):1738-1743. https://doi.org/ 10.1056/NEJM199506293322602

5. Andre M, Mounier N, Leleu X, et al. Second cancers and late toxicities after treatment of aggressive non-Hodgkin lymphoma with the ACVBP regimen:a GELA cohort study on 2837 patients. Blood. 2004;103(4): 1222-1228.

6. Hutchins LF, Unger JM, Crowley JJ, Coltman CA Jr, Albain KS. Underrepresentation of patients 65 years of age or older in cancertreatment trials. N Engl J Med. 1999;341(27):2061-2067. https://doi. org/10.1056/NEJM199912303412706

7. van Dalen EC, van der Pal HJ, Caron HN, Kremer LC. Different dosage schedules for reducing cardiotoxicity in cancer patients receiving anthracycline chemotherapy. Cochrane Database Syst Rev. 2009;4: CD005008. https://doi.org/10.1002/14651858.CD005008.pub3

8. van Dalen EC, Caron HN, Dickinson HO, Kremer LC. Cardioprotective interventions for cancer patients receiving anthracyclines. Cochrane Database Syst Rev. 2008;2:CD003917. https://doi.org/10.1002/ 14651858.CD003917.pub3

9. Swenson CE, Bolcsak LE, Batist G, et al. Pharmacokinetics of doxorubicin administered i.v. As Myocet (TLC D-99; liposome-encapsulated doxorubicin citrate) compared with conventional doxorubicin when given in combination with cyclophosphamide in patients with metastatic breast cancer. Anticancer Drugs. 2003;14(3):239-246. https://doi. org/10.1097/01.cad.0000060626.61556.da

10. Ewer MS, Martin FJ, Henderson C, Shapiro CL, Benjamin RS, Gabizon AA. Cardiac safety of liposomal anthracyclines. Semin Oncol. 2004; 31(6 Suppl 13):161-181.

11. Allen TM, Martin FJ. Advantages of liposomal delivery systems for anthracyclines. Semin Oncol. 2004;31(6 Suppl 13):5-15.

12. Batist G, Ramakrishnan G, Rao CS, et al. Reduced cardiotoxicity and preserved antitumor efficacy of liposome-encapsulated doxorubicin and cyclophosphamide compared with conventional doxorubicin and cyclophosphamide in a randomized, multicenter trial of metastatic breast cancer. J Clin Oncol. 2001;19(5):1444-1454.

13. Luminari S, Montanini A, Caballero D, et al. Nonpegylated liposomal doxorubicin (MyocetTM) combination (R-COMP) chemotherapy in elderly patients with diffuse large B-cell lymphoma (DLBCL): results from the phase II EUR018 trial. Ann Oncol. 2010;21(7):1492-1499. https://doi.org/10.1093/annonc/mdp544

14. Zaja F, Tomadini V, Zaccaria A, et al. CHOP-rituximab with pegylated liposomal doxorubicin for the treatment of elderly patients with diffuse large B-cell lymphoma. Leuk Lymphoma. 2006;47(10):2174-2180. https://doi.org/10.1080/10428190600799946
15. Rigacci L, Mappa S, Nassi L, et al. Liposome-encapsulated doxorubicin in combination with cyclophosphamide, vincristine, prednisone and rituximab in patients with lymphoma and concurrent cardiac diseases or pre-treated with anthracyclines. Hematol Oncol. 2007; 25(4):198-203. https://doi.org/10.1002/hon.827

16. Lown B, Wolf M. Approaches to sudden death from coronary heart disease. Circulation. 1971;44(1):130-142.

17. Cheson BD. The international harmonization project for response criteria in lymphoma clinical trials. Hematol Oncol Clin North Am. 2007;21(5):841-854

18. Perez-Soler R, Smith TL, Cabanillas F. Central nervous system prophylaxis with combined intravenous and intrathecal methotrexate in diffuse lymphoma of aggressive histologic type. Cancer. 1986;57(5): 971-977.

19. Federico M, Dyer MJS, Caballero MD, Reilly C, Thiel E. The MYOCAN study. a phase II study of cyclophosphamide, oncovin, Myocet, and prednisone plus rituximab (R-COMP) in the treatment of elderly patients with diffuse large B-cell non-Hodgkin lymphoma (DLBCL). Blood. 2004;104: abs 4586.

20. Limat S, Demesmay K, Voillat L, et al. Early cardiotoxicity of the CHOP regimen in aggressive non-Hodgkin's lymphoma. Ann Oncol. 2003; 14(2):277-281.

21. Moreno M, Sancho J-M, Gardella S, et al. Doxorrubicina liposomal no pegilada en combinación con ciclofosfamida, vincristina, prednisona y rituximab en el tratamiento de linfomas no hodgkinianos: estudio de 26 pacientes. Med Clin (Barc). 2010;134(2):72-75. https://doi.org/ 10.1016/j.medcli.2009.05.042

22. Mian M, Wasle I, Gamerith G, et al. R-CHOP versus R-COMP: are they really equally effective? Clin Oncol. 2014;26(10):648-652. https://doi. org/10.1016/j.clon.2014.05.012

23. Visani G, Ferrara F, Alesiani F, et al. R-COMP 21 for frail elderly patients with aggressive B-cell non-Hodgkin lymphoma: a pilot study. Leuk Lymphoma. 2008;49(6):1081-1086. https://doi.org/10.1080/ 10428190802043853

24. Gimeno E, Sánchez-González B, Alvarez-Larrán A, et al. Intermediate dose of nonpegylated liposomal doxorubicin combination (R-CMyOP) as first line chemotherapy for frail elderly patients with aggressive lymphoma. Leuk Res. 2011;35(3):358-362. https://doi.org/10.1016/j. leukres.2010.07.024

25. Dell'Olio M, Scalzulli RP, Sanpaolo G, et al. Non-pegylated liposomal doxorubicin (Myocet $\AA$ ) in patients with poor-risk aggressive B-cell non-Hodgkin lymphoma. Leuk Lymphoma. 2011:52(7):1222-1229. https://doi.org/10.3109/10428194.2011.572321

26. Herrero J, Gómez-Codina J, Provencio M, et al. Biweekly regimen of nonpegylated liposomal doxorubicin with cyclophosphamide, vincristine, and prednisone plus rituximab (R-COMP-14) as primary treatment for diffuse large B-cell lymphoma (DLBCL): Long-term follow-up of a phase II study. J Clin Oncol. 2012;30(suppl): abs 8056.

27. lannitto E, Luminari S, Mammi C, et al. Non-Pegylated Lyposomal doxorubicin, cyclophosphamide, vincristine, prednisone and rituximab (R-COMP) as initial treatment for patients with splenic marginal zone lymphoma (SMZL). A GISL study. Blood. 2007;110: abs 1293.

28. Fridrik MA, Jaeger U, Petzer A, et al. Cardiotoxicity with rituximab, cyclophosphamide, non-pegylated liposomal doxorubicin, vincristine and prednisolone compared to rituximab, cyclophosphamide, doxorubicin, vincristine, and prednisolone in frontline treatment of patients with diffuse large B-. Eur J Cancer. 2016;58:112-121. https://doi.org/ 10.1016/j.ejca.2016.02.004

29. Sancho J-M, Gual F, Fernández-Alvarez R, et al. R-COMP vs. R-CHOP as first-line treatment for De Novo diffuse large B-cell lymphoma in patients older than 60 years: Preliminary results from a prospective randomized phase 2 study from the Spanish group Geltamo. Blood. 2016;128: abs 5387

30. Fields PA, Townsend W, Webb A, et al. De Novo treatment of diffuse large B-cell lymphoma with rituximab, cyclophosphamide, vincristine, gemcitabine, and prednisolone in patients with cardiac 
comorbidity: a United Kingdom National Cancer Research Institute Trial. J Clin Oncol. 2014;32(4):282-287. https://doi.org/10.1200/ JCO.2013.49.7586

31. Rashidi A, Oak E, Carson KR, Wagner-Johnston ND, Kreisel F, Bartlett NL. Outcomes with R-CEOP for R-CHOP-ineligible patients with diffuse large B-cell lymphoma are highly dependent on cell of origin defined by Hans criteria. Leuk Lymphoma. 2016;57(5):1191-1193. https://doi.org/10.3109/10428194.2015.1096356

\section{SUPPORTING INFORMATION}

Additional Supporting Information may be found online in the supporting information tab for this article.
How to cite this article: Luminari S, Viel E, Ferreri AJM, et al. Nonpegylated liposomal doxorubicin combination regimen in patients with diffuse large B-cell lymphoma and cardiac comorbidity. Results of the HEART01 phase II trial conducted by the Fondazione Italiana Linfomi. Hematological Oncology. 2017. https://doi.org/10.1002/hon.2425 